\title{
Network Analysis, and Experimental Validation to Uncover the Mechanism of the Four Compounds in Artemisia annua (Qing Hao) Antimalarial
}

yuping zhao ( $\nabla 18810084632 @ 163 . c o m$ )

China Academy of Chinese Medical Sciences

Xiaobo Zhang

China Academy of Chinese Medical Sciences

Haiyu Xu

China Academy of Chinese Medical Sciences

Ling Wang

China Academy of Chinese Medical Sciences

Luqi Huang

China Academy of Chinese Medical Sciences

\section{Research}

Keywords: Artemisia annua; antimalaria; bioinformatics; interactive mechanism; synergistic effect

Posted Date: April 7th, 2020

DOI: https://doi.org/10.21203/rs.3.rs-19055/v1

License: (c) (1) This work is licensed under a Creative Commons Attribution 4.0 International License. Read Full License 


\section{Abstract}

Background: Artemisinin is widely used to anti malaria, but the antimalaria mechanism and coordinative interaction of Artemisinin, Scopoletin, Arteannuin B and Artemisic acid are not clear.

Methods: Based on the existing of antimalarial drugs, the antimalaria targets of Artemisinin, Scopoletin, Arteannuin B and Artemisic acid were explored by molecular docking with the similarity theory of chemical structure, and then the the antimalaria mechanism of Scopoletin and its coordinative antimalaria interaction of the mixture with the three other ingredients.

Results: Then through using the text information excavation's method, the relevance proteins of antimalaria effect of Artemisinin were IL-6, ACHE, PC3, IPOB, CYC, TNF-a, UGT1A9, CASP3, XDH, IL-1 3 , VEGF, CAT, CREB, AMPK, UGT1A6, ADR, MAPK, COX2, LB24AB and CYP450. Meanwhile, the relevance proteins of Scopoletin were TNF-a, PI3K, IL-8, IL- 6, VEGF, IL-1ß, MAPK, CD4, SP2, CTNNB, CASP3, PR01400, IgE, IL-4, ICAM1, p38, STAT3, TLR4 and API4. But Arteannuin B and Artemisic acid had a little relevance with the above proteins. The interaction characteristic between TNF-a and Artemisia annua was the effect of the mixture of Artemisinin, Scopoletin, Arteannuin B and Artemisic acid was greater than Artemisinin, and the synergistic effect of the four elements was found in the progress of antimalaria.

Conclusion: Antimalarial target of Artemisia annua ingredients was explored with data mining methods, and the antimalarial effect of Scopoletin may be related to TNF. Combined application of the four elements could achieve the same antimalarial effect and reduce the clinical usage of Artemisinin and Scopoletin.

\section{Background}

Malaria is a major enemy of human life. Together with AIDS and cancer, it is listed by the World Health Organization as one of the world's three major death diseases.

Before the advent and promotion of artemisinin, about 400 million people worldwide were infected with malaria every year, and at least 1 million people died of this disease. Infections and deaths are concentrated in relatively poor sub-Saharan Africa. In 2016, according to incomplete statistics, 2.7 billion dollars was cost in malaria control and elimination efforts globally not only by governments of malaria endemic countries, but also international partners [1]. Antimalarial drugs mainly include quinolines (such as chloroquine, mefloquine, quinine, etc.) and antifolates (such as pyrimethamine, sulfadoxine, etc.). The use of these drugs has effectively controlled the global spread of malaria. But then Plasmodium falciparum produces varying degrees of resistance to almost all antimalarial drugs [2]. China was once one of the major countries plagued by malaria, and tremendous efforts were made by the Chinese government [3]. Unique chemical composition in traditional Chinese materia medica has significant biological activity in major diseases. Chinese medicine with clear active ingredients is rare, while Artemisia annua (Qing Hao) is typical chemical composition obtained by modern scientifically verified traditional Chinese materia medica. Victory was finally achieved, in 2014, malaria patients were 
controlled to 56 individiuals [4]. In 1990s, artemisinin were widely used in Thailand and other countries in Southeast Asia. Soon, artemisinin has been widely used and focused in Africa and the Americas [5]. Artemisinin monotherapy doesn't work obviously, while artemisinin-based combination therapy is also negative. Delayed parasite clearance would confuse the clinical, the same with other antimalarial drugs [6-8]. It means that artemisinin don not work. Plasmodium falciparum has developed resistance to artemisinin in the Greater Mekong region, including Cambodia, Laos, Myanmar, Thailand and Vietnam. The World Health Organization's 2011 global plan to curb artemisinin resistance [9]. The reason might mainly from the the artemisinin partner drug [10]. Artemisinin if combined with another drug with low drug resistance, would not delay parasite clearance. It remains completely possible to rely on artemisinin and its new partner drugs to eliminate malaria in areas where malaria is endemic [11]. The drug with low drug resistance must be a new one, for existing antimalarials show strong resistance. Scopoletin, Arteannuin B and Artemisic acid were considered because of their chemical structure is more similar to Artemisinin. Compounds that selectively act on two or more targets of interest in theory would perform more pharmacological action than single-target agents [12]. 'Polypharmacology' is known as a new terminology that followed by drug finder [13]. This topic uses the method of network pharmacology to carry out text mining and target prediction by recording the four components of artemisinin, artemisinin, artemisinic acid and sorghum lactone, which may have antimalarial effects in Artemisia annua L.

Molecular docking is an essential procedure to verify network pharmacology in structural molecular biology and computer-assisted drug design. Molecular docking could be used to perform virtual screening on chemical compounds, rank the results, and propose structural hypotheses of how the ligands inhibit the target, which is invaluable in lead optimization [14].

\section{Materials And Methods}

Reagents and materials

1-ethyl-3-(3-dimetylaminopropyl)-carbodiimide hydrochloride (EDC) was obtained from Shanghai Beinuo Biotechnology Co. Ltd. (Shanghai, China), N-hydroxysuccinimide (NHS) was obtained from Shanghai Dibai Biotechnology Co. Ltd. (Shanghai, China), Sodium acetate and dimethyl sulfoxide (DMSO) were obtained from Beijing Chemical Plant (Beijing, China), Ethanolamine hydrochloride was purchased from Shanghai Sanying Chemical Reagent Co. Ltd. (Shanghai, China), the protein TNF-a was provided by RD Biosciences (America), Artemisinin, Scopoletin, Arteannuin B and Artemisic acid were provided by Chengdu Ruifensi Biological Technology Co., Ltd. (Chengdu, China), Dulbecco's phosphate buffered saline PBS buffer (PH4.7) were freshly prepared.

Plasma sample preparation

Weigh accurately $40 \mathrm{mgEDC}$ and $10 \mathrm{mg} \mathrm{NHS}$, make up to $1 \mathrm{~mL}$ with distilled water, and dissolve. Rinse the two channels thoroughly and quickly inject the above solution. The injection time is 5 minutes and rinse with PBS buffer. 
$50 \mu \mathrm{g}$ of TNF-a protein was dissolved in $100 \mu \mathrm{L}$ of PBS, and $10 \mu \mathrm{L}$ of the above solution was taken in three portions and diluted with sodium acetate solutions having $\mathrm{pH}$ values of $5.5,6.0$, and 6.5 , so that the final concentration was $50 \mu \mathrm{g} / \mathrm{ml}$. Reduce the flow rate to $20 \mu \mathrm{L} / \mathrm{min}$ and rinse the left channel for 10 minutes to determine the optimal $\mathrm{pH}$ of sodium acetate.

After determining the optimal $\mathrm{pH}$ value, $1 \mathrm{M}$ ethanolamine hydrochloride was injected into the two channels for 10 minutes to complete the sample fixation.

The four chemical composition Scopoletin (A), Artemisinin(B), Artemisic acid (C) and Arteannuin B (D) were divided into 12 groups according to the combination of $A, B, C, D, A B, A C, A D, A B C, A B D, A C D, B C D$, and $A B C D$, and each group was set to 6 concentrations. The control group was PBS buffer at pH 7.4.

Accurately weigh $19.2 \mathrm{mg}$ of scutellaria lactone, $28.2 \mathrm{mg}$ of artemisinin, $24.8 \mathrm{mg}$ of artemisinin 2 and $23.4 \mathrm{mg}$ of artemisinin, respectively, and dissolve them in $1 \mathrm{~mL}$ of DMSO (dimethyl sulfoxide). The DMSO solution in each group was gradient diluted with PBS to a final concentration of $200 \mu \mathrm{M}, 66.7 \mu \mathrm{M}$, $22.2 \mu \mathrm{M}, 7.41 \mu \mathrm{M}, 2.47 \mu \mathrm{M}$.

Targets fishing

Known therapeutic targets for the treatment of malaria were obtained from the DrugBank database (http://www.drugbank.ca/, version, 4.3) [15]. Only the "antimalaria" was selected as the key word, and the drug-target interactions whose drugs are approved by the Food and Drug Administration, USA (FDA) for treating menstrual disorders. All target gene/ protein identifiers (IDs) were converted into the correspondinggene symbol/UniProtKB-Swiss-Prot IDto facilitate further data analyses. After removing redundant entries, 25 target genes corresponding to 15 known antimalarial drugs were retrieved.

Protein-protein interaction (PPI) data

PPI data were imported from the following PPI databases, including Human Annotated and Predicted Protein Interaction Database (HAPPI, http://bio.informatics. iupui.edu/HAPPI/, Version 31.2) [16]. Based on the PPI network database, an interaction network of Artemisia annua candidate target groups and known antimalarial drug target groups was constructed. The distribution of target nodes in metabolic pathways and the corresponding diseases are revealed. A direct interaction network of key nodes was established, and the network was divided into modules according to the functions of the nodes. According to the malaria pathway (ko05144: Malaria) in KEGG, candidate verification molecules closely related to the malaria pathway were selected from the key nodes.

Network construction and topological analysis

Compound-target (C-T), target-pathway (T-P), and target-disease (T-D) networks about malaria were constructed using the Cytoscape 3.2 software (https://cytoscape. org/download.html), a general bioinformatics software package for data integration and visualization of biological network (Bindea et al., 2009; Smoot et al., 2011). An interaction network of Artemisia annua candidate target genes with 
known antimalarial drug target genes was established, consisting of 85 nodes and 298 pairs of interactions. Calculate the topological characteristic value of each node in the network, and use the median of the topological characteristic value as the card value. A total of 32 key nodes are screened. A direct interaction network of key nodes is established, and the network is processed according to the node functions. The module is divided, and the malaria pathway in Kyoto Encyclopediaof Genesand Genomes (KEGG) (ko05144: Malaria) is compared, and candidate verification molecules closely related to the malaria pathway are selected from key nodes.

Molecular docking.

The molecular structure of CDK4, NFKB1, PIK3CG, MAPK1, TNF and ITGB2 protein targets (protein species is human) is searched in the database uniprot (http://www.uniprot.org/). The structures of scopolamine and artemisinic acid are downloaded from the pubchem database (https://pubchem.ncbi.nlm.nih.gov). Chemical composition and protein structure are dehydrated and hydrotreated. Molecular docking and figures were generated Discovery Studio Visualizer software.

Probe Kd determination.

Weigh accurately $40 \mathrm{mg}$ EDC and $10 \mathrm{mg} \mathrm{NHS}$, make up to $1 \mathrm{~mL}$ with distilled water, and dissolve. Rinse the two channels thoroughly and quickly inject the above solution. The injection time is 5 minutes and rinse with PBS buffer. The protein TNF-a immobilized on Grafted Sensor Chips. The compound monomers and combinations are divided into 12 groups (Table 1). Each group of samples was injected at a concentration from low to high, and a control group (PBS) was set between each concentration. Regression analysis when concentration curves are separated and approaching equilibrium. The dissociation constant $(\mathrm{Kd})$ and its maximum value (Bmax) were then calculated by fitting the titration curve to the single-site saturation binding equation $[\mathrm{Y}=\mathrm{Bmax} \times \mathrm{X} /(\mathrm{Kd}+\mathrm{X})]$ using the GraphPad Prism software (Graphpad software Incorporated, La Jolla, CA, USA). 
Table 1

The compound monomers and combinations

\begin{tabular}{|ll|}
\hline Group & compounds \\
\hline A & Scopoletin \\
\hline B & Artemisinin \\
\hline C & Artemisic acid \\
\hline D & Arteannuin B \\
\hline AB & Scopoletin ;Artemisinin \\
\hline AC & Scopoletin; Artemisic acid \\
\hline AD & Scopoletin; Arteannuin B \\
\hline ABC & Scopoletin; Artemisinin ;Artemisic acid \\
\hline ABD & Scopoletin; Artemisinin; Arteannuin B \\
\hline ACD & Scopoletin; Artemisic acid; Arteannuin B \\
\hline BCD & Artemisinin ;Artemisic acid; Arteannuin B \\
\hline ABCD & Scopoletin; Artemisinin; Artemisic acid; Arteannuin B \\
\hline
\end{tabular}

\section{Results}

Compound-target network construction

162 diseases related to four chemical components in Artemisia annua were discovered. Through artificial noise reduction, the top 5 representative diseases with frequency of occurrence were extracted as malaria (50), cerebral malaria (7), falciparum malaria (4), visceral leishmaniasis (2), and systemic lupus erythematosus (1), Fig. 1. 15 known antimalarial drugs retrieved based on DrugBank database, Table 2. There are 93 targets with similar structures of scopolide and 15 known antimalarial drugs (Similar score than 0.7). Artemisinic acid has 32 targets with similar structures with 15 known antimalarial drugs (Similar score than 0.7).

Clustering analysis

The key node interaction network is divided into three functional modules. The first functional module mainly involves immune-related pathways, the second functional module mainly involves multiple infectious diseases, and the third functional module is related to drug metabolism and tumor pathways; Comparing KEGG's malaria pathway (ko05144: Malaria), Fig. 2. The four key nodes involved in the above four pathways: $T$ cell receptor signaling pathway, Toll-like receptor signaling pathway, TNF signaling 
pathway, Natural killer cell mediated cytotoxicity are all in the malaria pathway Important links, so the joint nodes participating in these four pathways can be used as candidate verification objects.

Table 2

Known antimalarial drugs

\begin{tabular}{|ll|}
\hline Drug ID & Name \\
\hline DB00254 & Doxycycline \\
\hline DB00908 & Quinidine \\
\hline DB00358 & Mefloquine \\
\hline DB01611 & Hydroxychloroquine \\
\hline DB00608 & Chloroquine \\
\hline DB00613 & Amodiaquine \\
\hline DB01103 & Quinacrine \\
\hline DB01087 & Primaquine \\
\hline DB00468 & Quinine \\
\hline DB04877 & Voacamine \\
\hline DB00440 & Trimethoprim \\
\hline DB01218 & Halofantrine \\
\hline DB00205 & Pyrimethamine \\
\hline DB01131 & Proguanil \\
\hline DB01117 & Atovaquone \\
\hline
\end{tabular}

Molecular docking

Scopolide has suitable docking sites with TNF, NFKB1, and PIK3CG, and the docking scores are 77.9576, 57.8491, and 50.2248 respectively, while no suitable docking site with CDK4, MAPK1. There is no suitable docking site for Artemisinic acid with ITGB2 (Table 3, Fig. 3). 
Table 3

Docking score of Scopolide and artemisinic acid with the targets

\begin{tabular}{|lll|}
\hline Compounds & Tagets & Docking Score \\
\hline Scopolide & CDK4 & - \\
\hline Scopolide & NFKB1 & 57.8491 \\
\hline Scopolide & PIK3CG & 50.2248 \\
\hline Scopolide & MAPK1 & - \\
\hline Scopolide & TNF & 77.9576 \\
\hline artemisinic acid & ITGB2 & - \\
\hline
\end{tabular}

Experimental validation of key targets

TNF has good binding with scopolide, suggesting that the antimalarial effect of scopolide may be related to TNF. The binding of TNF to artemisinin, artemisinin B, and artemisinic acid is poor, but the combination of 4 components of artemisinin, artemisinin B, artemisinic acid, and stigmalactone has good binding to TNF, suggesting Combined application of 4 ingredients may achieve antimalarial effects by acting on TNF (Table 4, Fig. 4). 
Table 4

Equilibrium dissociation constant KD for each group

\begin{tabular}{|llll|}
\hline Group & KD(M) & Est. error & Bmax Signal (URIU) \\
\hline A & $4.05 \times 10^{-6}$ & $2.00 \times 10^{-6}$ & 42.0 \\
B & $2.01 \times 10^{-4}$ & $1.45 \times 10^{-5}$ & 38.7 \\
\hline C & $8.91 \times 10^{-5}$ & $4.07 \times 10^{-7}$ & 72.3 \\
\hline D & $2.93 \times 10^{18}$ & $1.39 \times 10^{-7}$ & $2.78 \times 10^{17}$ \\
AB & $1.35 \times 10^{-3}$ & $8.08 \times 10^{-6}$ & 46.7 \\
\hline AC & $1.25 \times 10^{-4}$ & $3.97 \times 10^{-6}$ & 33.7 \\
\hline AD & $3.29 \times 10^{-4}$ & $1.72 \times 10^{-6}$ & 15.4 \\
\hline ABD & $1.20 \times 10^{-4}$ & $1.61 \times 10^{-6}$ & 12.7 \\
\hline ABC & $2.69 \times 10^{-4}$ & $8.38 \times 10^{-7}$ & 25.8 \\
\hline ACD & $1.59 \times 10^{20}$ & $4.91 \times 10^{-7}$ & $8.36 \times 10^{18}$ \\
\hline BCD & $2.80 \times 10^{-5}$ & $1.78 \times 10^{-5}$ & 10.1 \\
\hline ABCD & $2.17 \times 10^{-5}$ & $5.04 \times 10^{-5}$ & 12.5 \\
\hline
\end{tabular}

\section{Discussion}

The sesquiterpene compounds represented by artemisinin are the most active field in artemisia annua. At present, nearly 61 sesquiterpenoids have been isolated from Artemisia annua, mainly artemisinin compounds, including artemisinic acid, artemisinol, artemisinin ether and artemisinin. Artemisinin is a sesquiterpene lactone containing an endoperoxy bridge structure and is the main component of antimalarial. Research data show that artemisinin and artemisinin can be converted to artemisinin in the original plants $[17,18]$. Summarized the biosynthetic routes of artemisinin, among which there were 9 total synthetic routes and 5 semi-synthetic routes. The method of synthesizing artemisinin by the total synthesis method has a long route, high cost, and low total yield, up to $10 \%$. Artemisinic acid is one of the main components of sesquiterpenes in Artemisia annua plants and an important precursor of artemisinin synthesis. Studies by Tu Yu et al. Showed that the young plants of Artemisia annua contained a large amount of artemisinic acid and lacked artemisinin. It is speculated that sesquiterpenoids such as artemisinin were converted from artemisinic acid. Researchers like Levesque $F$ and others used synthetic biology to successfully produce artemisinin using genetically engineered yeast, and synthesized artemisinin [19]. Scopolide has strong water solubility and stability relative to artemisinin, etc., and has 
pharmacological activity that reflects the efficacy of traditional artemisinin. Studies have shown that scopolide has a certain antimalarial effect, and it has certain effects with artemisinin. Synergy. With the rapid development of chemical genomes and pharmacological technologies, a large number of potential targets and massive biological activity data have emerged. However, with the accumulation of redundant data, simple analysis methods can no longer satisfy high-throughput large-scale data. Analytical needs [20]. The rapid development of chemical informatics has just met the requirements of big data processing and information extraction tasks that chemical genomics urgently needs to solve. Chemical informatics mainly studies how to properly select the diversity of compound libraries, how to characterize drug molecular characteristics, how to measure the differences between different molecules, how to identify drug-like molecules, molecular structure and biological performance relationships, and how to develop corresponding computer software and hardware This includes research tasks and content of chemometrics and computational chemistry [21]. An important application of the cheminformatics method in the post-genomic era is to predict the potential targets of small molecule compounds based on existing biological and chemical information, and to explain their mechanism of action to accelerate the development of drugs. The prediction of drug targets is of great significance to the evaluation of early drug molecules and the new use of old drugs, but due to the limitations of throughput, accuracy and cost, it is difficult to widely apply experimental methods. As a fast and low-cost method, the development of computer-aided target prediction algorithms is receiving more and more attention. According to different research strategies, prediction of drug targets based on chemoinformatics can be divided into three categories: prediction based on ligand characteristics, prediction based on protein structural characteristics, and prediction based on data mining methods $[22,23]$. According to the target prediction, it is found that the possible target of scopolide is CDK4, NFKB1, PIK3CG, MAPK1, TNF, and the possible target of artemisinic acid is ITGB2. Cyclin-dependent kinase (CDK) is a type of serine / threonine (Thr) kinase, which is an important signal transduction molecule in the cell and CDK-cyclin formed by cyclin Complex, involved in cell growth, proliferation, dormancy, or entering apoptosis. During the cell cycle, cyclins are periodically and continuously expressed and degraded, and are respectively bound to the CDKs activated by them transiently. Through the activity of CDKs, they catalyze the phosphorylation of different substrates to achieve the promotion and transformation of different phases of the cell cycle. The CDK family includes $1-13$, cyclin is divided into $A-L$, and different CDKs are connected to different cyclins. CDK4/6-specific activation is closely related to the proliferation of some tumors. $\mathrm{Rb}$ is present in approximately $80 \%$ of human tumors, and abnormalities in the cyclin D-CDK4/6-INK4-Rb pathway are common [24]. Cyclin-dependent kinase (CDK) is a type of serine/threonine (Thr) kinase, which is an important signal transduction molecule in the cell and CDK-cyclin formed by cyclin Complex, involved in cell growth, proliferation, dormancy, or entering apoptosis. During the cell cycle, cyclins are periodically and continuously expressed and degraded, and are respectively bound to the CDKs activated by them transiently. Through the activity of CDKs, they catalyze the phosphorylation of different substrates to achieve the promotion and transformation of different phases of the cell cycle. CDK4/6-specific activation is closely related to the proliferation of some tumors. $\mathrm{Rb}$ is present in approximately $80 \%$ of human tumors, and abnormalities in the cyclin D-CDK4/6-INK4-Rb pathway are common. It is characterized by: (1) p16INK4a gene deletion, point mutation, or DNA methylation leading to inactivation 
of p16INK4a; (2) CDK4 gene amplification or point muta T cells can induce other cells to activate or interfere with lysis. CD3, CD4, and CD8 cells are involved in T cell transcription of activation signals. TollLike receptors (TLRs) play an important role in the identification of invading pathogenic microorganisms in early innate immunity. These evolutionarily conserved receptors are structurally homologous to the Drosophila Toll protein family, recognizing highly conserved structural motifs (Motifs)-pathogenassociated molecular patterns expressed only on pathogenic microorganisms molecular pattern (PAMP). TLRs are stimulated by PAMPs to initiate a signal cascade including some proteins, leading to the activation of the transcription factor NF-kB, inducing the secretion of pro-inflammatory cytokines and effector cytokines directly involved in the adaptive immune response. TTGB2: Integrin B2, CD18, is an important member of the integrin family of adhesion molecules. It binds to different integrin subunits to form the leukocyte adhesion receptor group. It is mainly expressed in white blood cells, and its ligands are TCAM, iC3b, and fibrinogen. Its cytoplasmic region is linked to a variety of cytoskeleton proteins and is involved in signal transduction. Its genetic defects lead to leukocyte adhesion deficiency syndrome. ITGB2 mainly exists in Natural killer cell mediated cytotoxicity pathway. In this project, the biological macromolecule interaction instrument was used to verify the binding of the target protein TNF with scopolide, which had the highest score in the docking experiment. The binding effect was good. It was confirmed that scopolide could act on TNF and participate in its corresponding functions. It was confirmed that scorolactone has antimalarial effect. However, at the same time, the binding rates of artemisinin, artemisinin B, artemisinic acid and TNF are very low, which may be because these three compounds do not act on TNF. It also shows that the antimalarial effect of artemisinin may be antimalarial The malaria mechanism is different. The combination of the four ingredients with TNF is also very high, indicating that the four ingredients have a synergistic effect. The combined use of the four ingredients can reduce the use of artemisinin and scopolamine and achieve the same antimalarial effect. The characteristics of multi-component, multi-target, and synergistic effects of traditional Chinese medicine preparations. Many experiments have proved that TNF has a certain killing effect on Plasmodium. TNF must be assisted by certain factors (or factors) in the body in order to exert its ability to damage Plasmodium, that is, TNF is not a terminal effector that directly kills Plasmodium [46]. At present, it is thought that the immunoprotective mechanism of TNF may have the following types: (1) Enhance the phagocytosis function of phagocytic cells: The study found that neutrophils were treated with different doses of TNF for 30 minutes and were bred with Plasmodium falciparum, and the neutrophils were found. The phagocytosis of each stage of Plasmodium was strengthened, and the extent of the increase was positively correlated with the dose of TNF within a certain range; (2)Via reactive oxygen mediator: When TNF and macrophages were co-incubated for $30 \mathrm{~min}$, the release of reactive oxygen species (ROS from macrophages) was detected). And this ROS can kill Plasmodi.

\section{Conclusion}

Based on existing literature, data mining methods were used to find the targets of the antimalarial active ingredients artemisinin and scopolamine in Artemisia annua: 20 proteins related to artemisinin's antimalarial: IL-6, ACHE, PC3, IPOB, CYC, TNF-a, UGT1A9, CASP3, XDH, IL-1ß, VEGF, CAT, CREB, AMPK, 
UGT1A6, ADR, MAPK, COX2, LB24AB, CYP450. 19 scopolamine antimalarial related proteins: TNF-a, PI3K, IL-8, IL-6, VEGF, IL-1ß, MAPK, CD4, SP2, CTNNB, CASP3, PR01400, IgE, IL-4, ICAM1, P38, STAT3, TLR4, API4, artemisinic acid and artemisinin no related proteins.

Applying the principle of structural similarity of compounds to target prediction based on 15 known antimalarial drugs and their associated 25 target genes, artemisinin, artemisinin, artemisinic acid, and scopolamine Four component candidate targets: scopolide candidate targets CDK4, NFKB1, PIK3CG, MAPK1, TNF; artemisinin candidate target ITGB2, artemisinin and artemisinin B did not predict related targets.

TNF is poorly bound to artemisinin, artemisinin B, and artemisinic acid, but the combination of the four components of artemisinin, artemisinin B, artemisinic acid, and stigmalactone has good binding to TNF, Suggesting that the combined application of the four ingredients may achieve anti-malarial effects by acting on TNF, reconfirming the characteristics of multi-component, multi-target, and synergistic effects of traditional Chinese medicine preparations, to further rationally apply artemisia annua herbs and reduce artemisinin drugs Antimalarial resistance and the development of new drugs based on effective sites provide ideas.

\section{Abbreviations}

NHS: N-hydroxysuccinimide; DMSO: dimethyl sulfoxide; PPI: protein-protein interaction; KEGG: kyoto encyclopediaof genesand genomes; Thr: threonine; CDK: cyclin-dependent kinase; PAMP: pathogenic microorganisms molecular pattern.

\section{Declarations}

Acknowledgements

We thank Dr. Zhenyu Zhao for his critical reading and the key project at central government level for providing the support to the case investigation.

Authors' contributions

Z-yP, W-L, H-IQ integrating pharmacokinetics study, Z-xB, X-hY, network analysis, and experimental validation to research the antimalaria mechanism of Artemisinin, Scopoletin, Arteannuin B and Artemisic acid. Z-yP and H-IQ write the manuscript.

\section{Funding}

This work was funded by Key project at central government level: The ability establishment of sustainable use for valuable Chinese medicine resources, grant No. [2060302].

Availability of data and materials

Page $12 / 18$ 
All relevant data are included in this report.

Ethics approval and consent to participate

There are no ethics statement in this paper.

Consent

Not applicable.

Competing interests

The authors declare that they have no competing interests.

Author details

1. China Academy of Chinese Medical Sciences, Beijing 100700, China;

2. State Key Laboratory Breeding Base of Dao-di Herbs, National Resource Center for Chinese Materia Medica, China Academy of Chinese Medical Sciences, Beijing 100700, China;

3. Institute of Chinese Materia Medica, China Academy of Chinese Medical Sciences, Beijing 100700, China.

\section{References}

1. Organization WH: World malaria report 2015. World Health Organization; 2016.

2. Sá JM, Twu O, Hayton K, Reyes S, Fay MP, Ringwald P, Wellems TE: Geographic patterns of Plasmodium falciparum drug resistance distinguished by differential responses to amodiaquine and chloroquine. Proceedings of the National Academy of Sciences 2009, 106:18883-18889.

3. Hsiang MS, Hwang J, Tao AR, Liu Y, Bennett A, Shanks GD, Cao J, Kachur SP, Feachem RG, Gosling RD: Mass drug administration for the control and elimination of Plasmodium vivax malaria: an ecological study from Jiangsu province, China. Malaria journal 2013, 12:383.

4. Zhang L, Zhou S, Feng J, Fang W, Xia Z: Malaria situation in the People's Republic of China in 2014. Chin J Parasitol Parasit Dis 2015, 33:319-326.

5. Mclntosh H, Olliaro P: Artemisinin derivatives for treating severe malaria. Cochrane Database of Systematic Reviews 1998.

6. Jambou R, Legrand E, Niang M, Khim N, Lim P, Volney B, Ekala MT, Bouchier C, Esterre P, Fandeur T:

Resistance of Plasmodium falciparum field isolates to in-vitro artemether and point mutations of the SERCA-type PfATPase6. The Lancet 2005, 366:1960-1963.

7. Vijaykadga S, Rojanawatsirivej C, Cholpol S, Phoungmanee D, Nakavej A, Wongsrichanalai C: In vivo sensitivity monitoring of mefloquine monotherapy and artesunate-mefloquine combinations for the 
treatment of uncomplicated falciparum malaria in Thailand in 2003. Tropical Medicine \& International Health 2006, 11:211-219.

8. Noedl H, Se Y, Schaecher K, Smith BL, Socheat D, Fukuda MM: Evidence of artemisinin-resistant malaria in western Cambodia. New England Journal of Medicine 2008, 359:2619-2620.

9. W Muregi F, G Kirira P, Ishih A: Novel rational drug design strategies with potential to revolutionize malaria chemotherapy. Current medicinal chemistry 2011, 18:113-143.

10. Gil JP, Krishna S: pfmdr1 (Plasmodium falciparum multidrug drug resistance gene 1): a pivotal factor in malaria resistance to artemisinin combination therapies. Expert review of anti-infective therapy 2017, 15:527-543.

11. Wang J, Xu C, Liao FL, Jiang T, Krishna S, Tu Y: A Temporizing Solution to "Artemisinin Resistance". New England Journal of Medicine 2019, 380:2087-2089.

12. Hopkins AL: Network pharmacology. Nature biotechnology 2007, 25:1110.

13. Paolini GV, Shapland RH, van Hoorn WP, Mason JS, Hopkins AL: Global mapping of pharmacological space. Nature biotechnology 2006, 24:805.

14. Morris GM, Lim-Wilby M: Molecular docking. In Molecular modeling of proteins. Springer; 2008: 365382

15. Wishart DS, Knox C, Guo AC, Cheng D, Shrivastava S, Tzur D, Gautam B, Hassanali M: DrugBank: a knowledgebase for drugs, drug actions and drug targets. Nucleic acids research 2007, 36:D901D906.

16. Chen JY, Mamidipalli S, Huan T: HAPPI: an online database of comprehensive human annotated and predicted protein interactions. BMC genomics 2009, 10:S16.

17. Nair M, Basile D: Bioconversion of arteannuin B to artemisinin. Journal of natural products 1993, 56:1559-1566.

18. Wu W, Yuan M, Zhang Q, Zhu Y, Yong L, Wang W, Qi Y, Guo D: Chemotype-dependent metabolic response to methyl jasmonate elicitation in Artemisia annua. Planta medica 2011, 77:1048-1053.

19. Lévesque F, Seeberger PH: Continuous-flow synthesis of the anti-malaria drug artemisinin. Angewandte Chemie International Edition 2012, 51:1706-1709.

20. Yang $\mathrm{H}$, Li X: Chemical proteomics and discovery of drug targets. Yao xue xue bao=Acta pharmaceutica Sinica 2011, 46:877-882.

21. Gasteiger J: The scope of chemoinformatics. Handbook of Chemoinformatics: From Data to Knowledge in 4 Volumes 2003:3-5.

22. Koutsoukas A, Simms B, Kirchmair J, Bond PJ, Whitmore AV, Zimmer S, Young MP, Jenkins JL, Glick $\mathrm{M}$, Glen RC: From in silico target prediction to multi-target drug design: current databases, methods and applications. Journal of proteomics 2011, 74:2554-2574.

23. Zheng M, Liu X, Xu Y, Li H, Luo C, Jiang H: Computational methods for drug design and discovery: focus on China. Trends in pharmacological sciences 2013, 34:549-559. 
24. Malumbres M, Barbacid M: Milestones in cell division: to cycle or not to cycle: a critical decision in cancer. Nature Reviews Cancer 2001, 1:222.

\section{Figures}

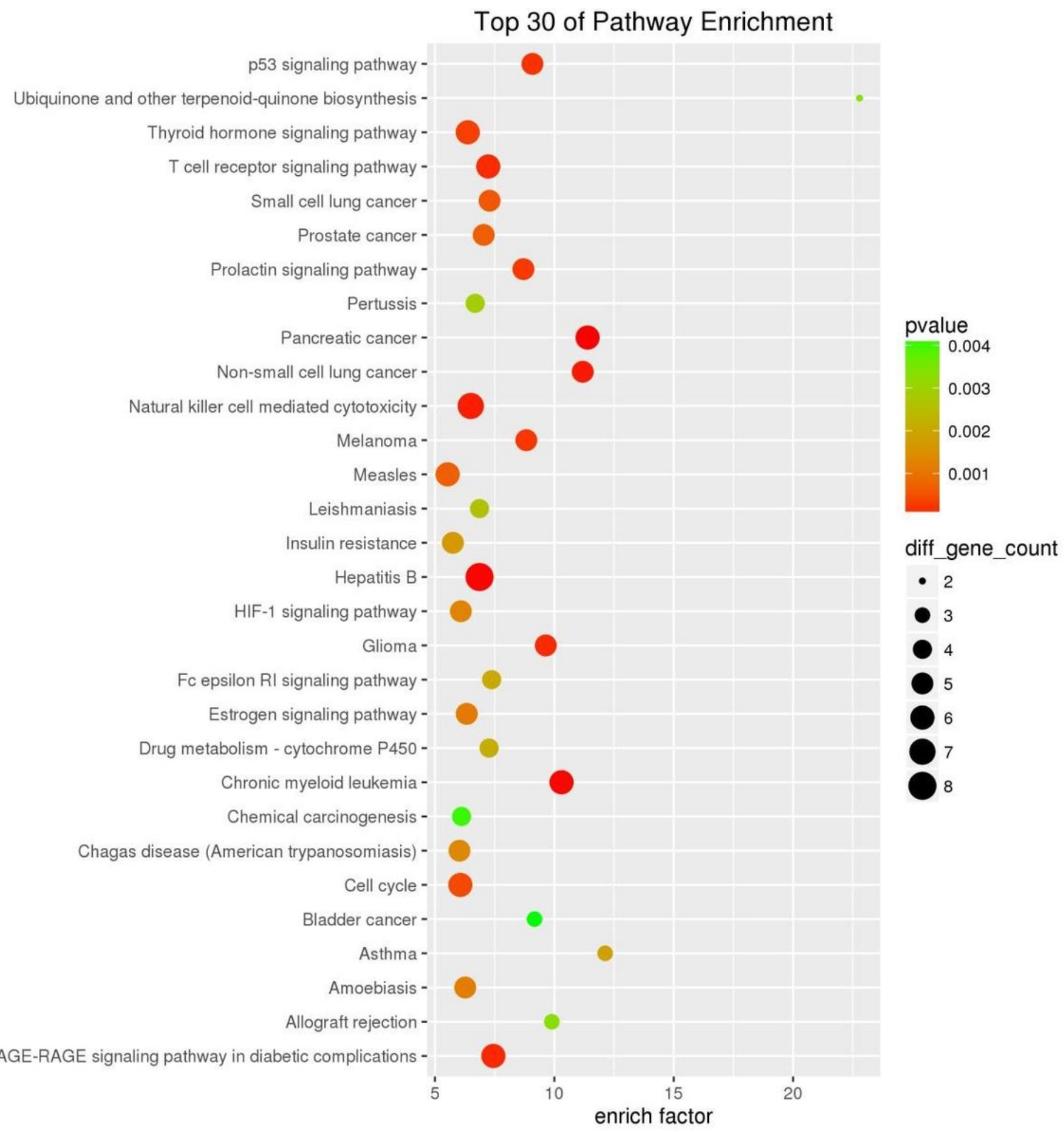

Figure 1 
The above candidate targets significantly participate in multiple immune-related pathways, such as Fc epsilon RI signaling pathway, T cell receptor signaling pathway, and Natural killer cell mediated cytotoxicity; and multiple infectious disease-related pathways, such as Hepatitis B (hepatitis B), Leishmaniasis (leish (Mann disease), Pertussis, Amoebiasis, Measles, Toxoplasmosis and Hepatitis C.

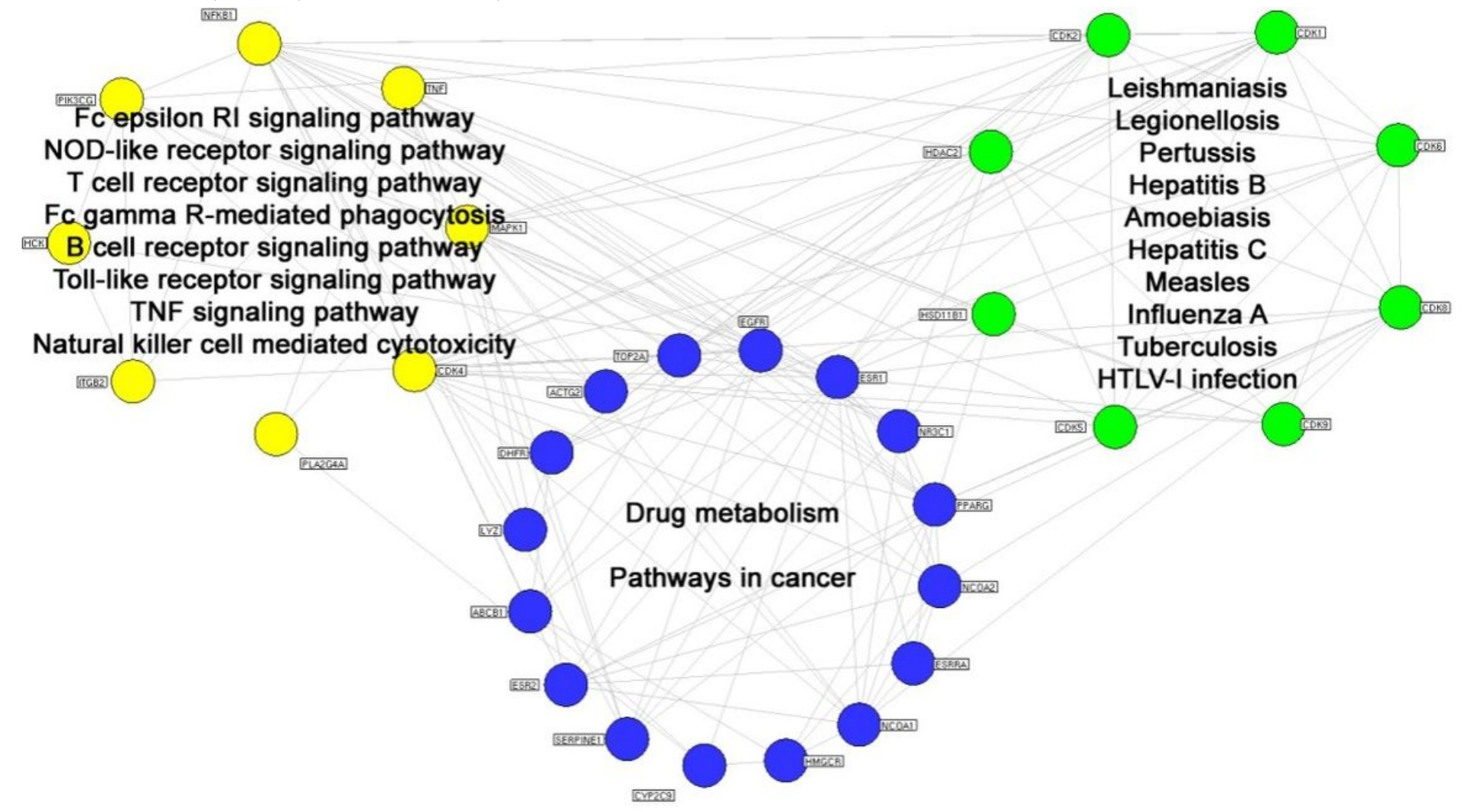

Figure 2

Establishment of an interaction network between Artemisia annua candidate target genes and known antimalarial drug target genes, consisting of 85 nodes and 298 pairs of interactions. 


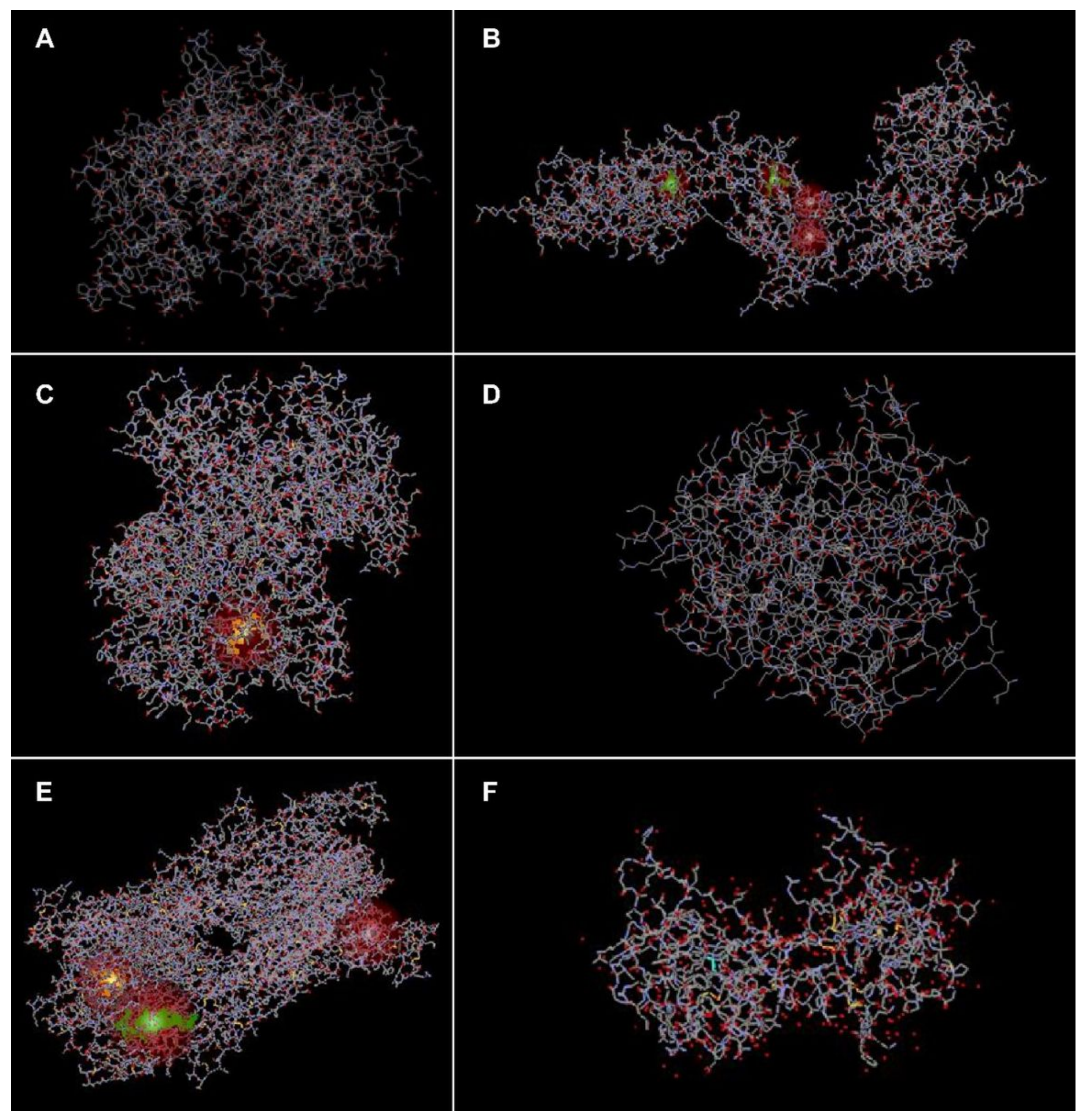

\section{Figure 3}

Molecular Docking by Discovery Studio. A Scopolide with CDK4; B Scopolide with NFKB1; C Scopolide with PIK3CG; D Scopolide with MAPK1; E Scopolide with TNF; F r Artemisinic acid with ITGB2 


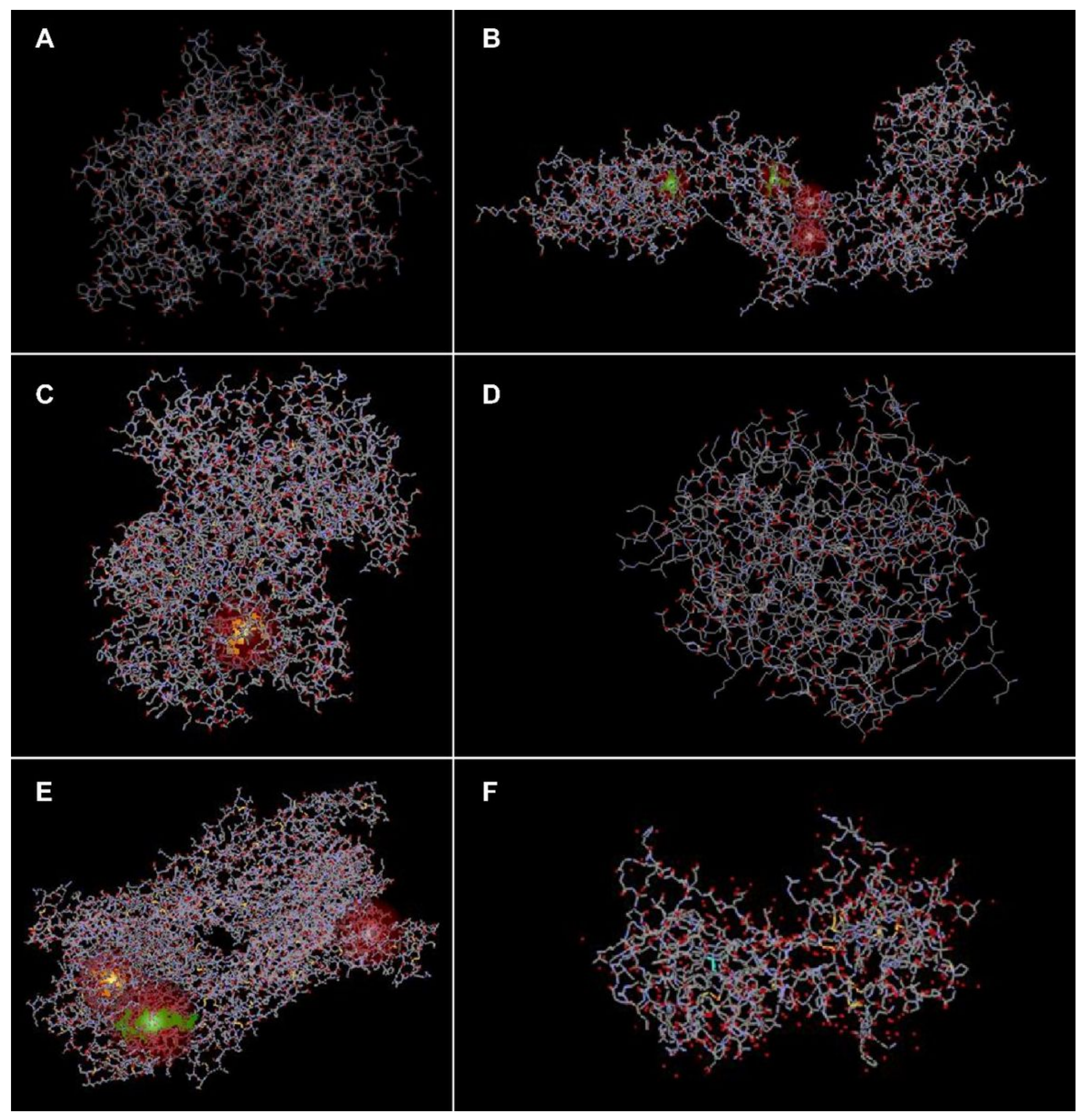

\section{Figure 4}

The dissociation constant $(\mathrm{Kd})$ of each group. Grouping is the same as in Table 1. 